\title{
DYNAMIC VEHICLE TRAFFIC MANAGEMENT SYSTEM
}

\author{
Supriya Kamoji ${ }^{1}$, Aswathi Nambiar ${ }^{2}$, Karishma Khot ${ }^{3}$, Ravi Bajpai ${ }^{4}$ \\ ${ }^{1}$ Assistant Professor, Computer Engineering, Fr. Conceicao Rodrigues College of Engineering, Maharashtra, India \\ ${ }^{2}$ B.E. student, Computer Engineering, Fr. Conceicao Rodrigues College of Engineering, Maharashtra, India \\ ${ }^{3}$ B.E. student, Computer Engineering, Fr. Conceicao Rodrigues College of Engineering, Maharashtra, India \\ ${ }^{4}$ B.E. student, Computer Engineering, Fr. Conceicao Rodrigues College of Engineering, Maharashtra, India
}

\begin{abstract}
Traffic congestion on city road networks is one of the main issues to be addressed by today's traffic management schemes. The frequent traffic jams at major junctions call for an efficient traffic management system in place. The image sequences from a camera are analyzed using edge detection technique, object counting method and queue length estimation to obtain the most efficient technique. Subsequently, the number of vehicles at the intersection is evaluated and traffic is efficiently managed. The paper also proposes to implement a real-time emergency vehicle detection system. In case an emergency vehicle is detected, the lane is given priority over all the others. Using image-processing operations to calculate traffic density is cost effective as cameras are cheaper and affordable devices compared to any other devices such as sensors.
\end{abstract}

Keywords: Edge detection, Object counting, vehicle queue length, traffic management, image processing.

\section{INTRODUCTION}

In Today's Scenario it is fact that, number of vehicles is increasing exponentially, but infrastructure for transportation is not sufficient to satisfy their needs. Due to this, valuable time is being lost every day. This also leads to huge economic problems. Main problem occurs when this traffic congestion costs life of someone. It should not be surprising that traffic congestion affects almost all emergency vehicles, which can be too much hazardous to affected people. Current traffic control techniques involving magnetic loop detectors buried in the road, infra-red and radar sensors on the side provide limited traffic information and require separate systems for traffic counting and for traffic surveillance. Inductive loop detectors do provide a cost effective solution, however they are subject to a high failure rate when installed in poor road surfaces, decrease pavement life and obstruct traffic during maintenance and repair. Infrared sensors are affected to a greater degree by fog than video cameras and cannot be used for effective surveillance. In contrast, video-based systems offer many advantages compared to traditional techniques. They provide more traffic information, combine both surveillance and traffic control technologies, are easily installed, and are scalable with progress in image processing techniques. This paper tries to evaluate the process and advantages of the use of image processing for traffic control. Implementation of this project will eliminate the need of traffic personnel at various junctions for regulating traffic[1]. Thus, the use of this technology is valuable for the analysis and performance improvement of road traffic.

The traffic lights are embedded, it is not reliable as the traffic flow can never be interpreted and it's proved to be a non-reliable technology. It is based on 2 minute time interval, the vehicles move at a normal speed when the light is green for a certain interval of time(e.g. 2 minute), but come to a stop when it turns red after the interval. Sometimes when there are no vehicles in lane but still it's given green because traffic signals change depending upon time interval [2] . On the other hand, image processing may be used to dynamically release traffic, depending on which, lane is congested irrespective of the time based system.

\section{OVERVIEW OF EXISTING SYSTEM}

\subsection{Time Based}

In order to remove the weakest link (i.e. human) in our system, an automatic traffic management scheme is suggested. This system includes simple three color traffic signal, which we see every day. Generally for each lane 120 seconds of green light is on. Before green light, yellow light flashes for 20 second, signifying to start your vehicle and be ready to go. For all the time red light is on, ordering each vehicle to stop. This system cannot identify emergency vehicle, it treats normal car and ambulance same way. So there are chances of delay. Besides unnecessary waiting time and congestion is increased [3]. 


\subsection{Embedded Systems}

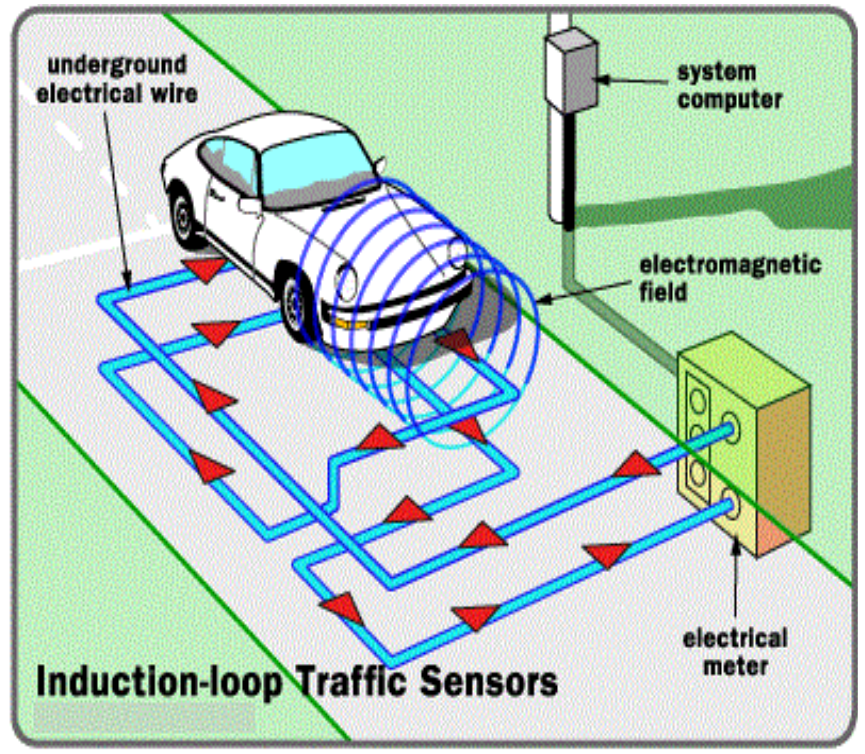

Fig 2.1: IR sensor system

Fig 2.1 shows that system uses IR sensors, AVR-32 microcontroller with programmable flash memory and built in 8-channels ADC. IR (i.e. Infrared) sensor is programmed to detect emergency vehicle and microcontroller is designed in such a way to give red signal to all other lane but one with emergency vehicle. Limitations: Here IR sensors are used, due to various climate conditions, IR sensors may need to keep in safe place or a strong box. Hence it is not advisable to implement this system [4].

\subsection{Wireless Sensor Networks}

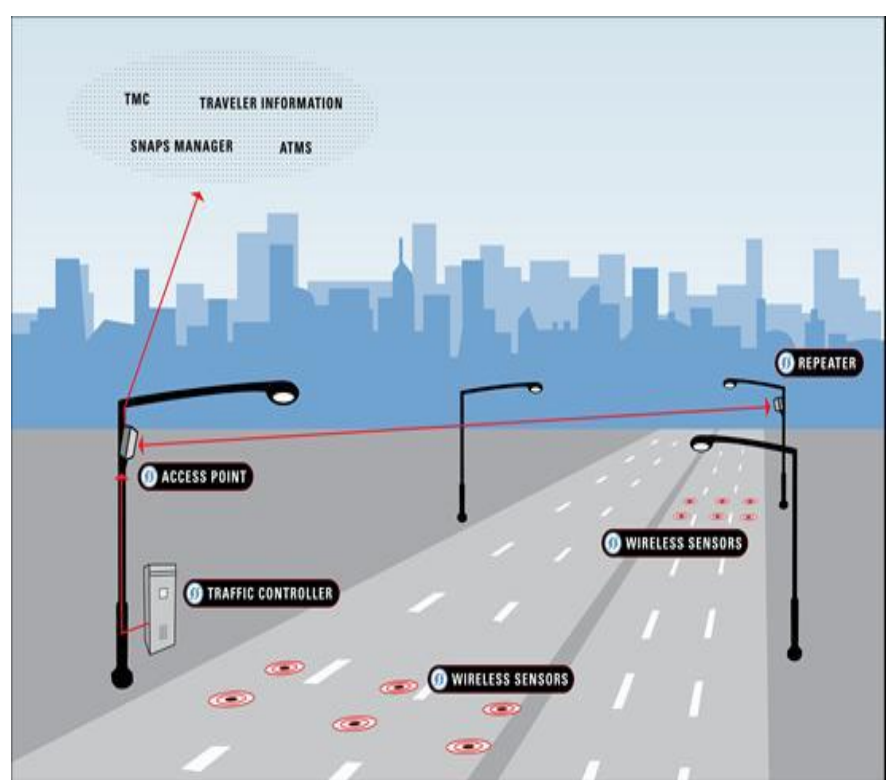

Fig 2.2: Wireless sensors on a lane.

Wireless Sensor Network shown in fig 1.2 is being used as communication infrastructure in the proposed traffic light controller. System uses fuzzy logic to define direction of emergency vehicle. Central monitoring system collects all information and gives appropriate response. Limitations:
Communication in Wireless Sensor Network is still a research field. Data exchange in between Sensor is not reliable [5].

\subsection{RFID Technology}

System includes Active RFID tag, Wireless Router, Wireless Coordinator, GSM modems, and Monitoring Station software. Wireless devices collects data from Active RFID tags, these devices are mounted at roadside. Monitoring station collects all data through GSM, and reply to corresponding traffic signal. Limitations: Involvement of various communication devices makes implementation costly. Wireless communications have their own drawbacks. A Monitoring Station needs to be setup [6].

\section{PROPOSED SYSTEM}

\subsection{Architecture}

A camera is fixed on polls or other tall structures to overlook the traffic scene. The traffic scenario at one of the checkpoint is as shown in fig 3.1.

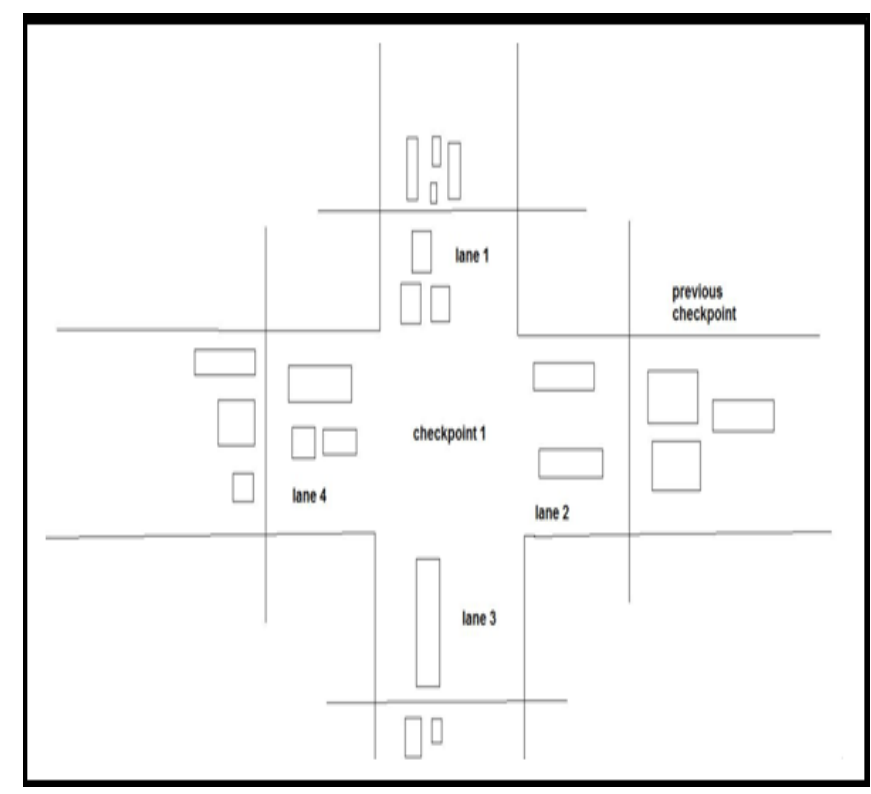

Fig 3.1.1 Traffic scenario at of one of the checkpoint

Consider each frame and Perform Edge Detection in order to highlight relevant edges of the objects. Check the previous checkpoint and calculate the sum of the objects/sum of the queue length on the two checkpoints. Compare the summation of the objects/queue length on individual lanes with the neighboring lanes. Then depending on the lane with maximum density of vehicles, time is allotted to each lane or the green signal is given to the lane with maximum density. 


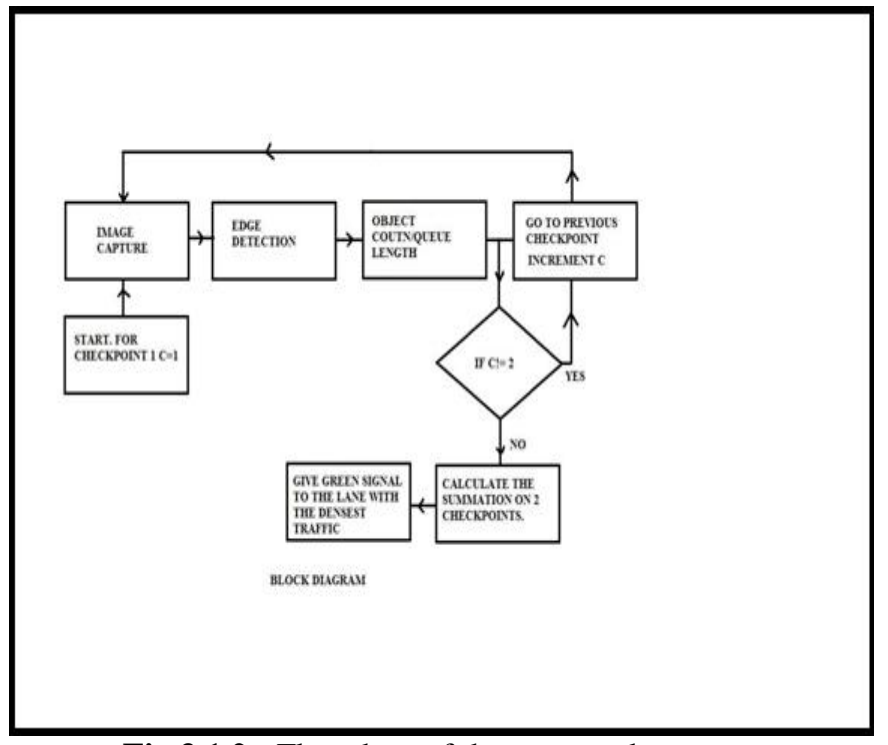

Fig 3.1.2: Flowchart of the proposed system

Proposed system is implemented using three different techniques.

1. Object count

2. Queue length estimation

3. Combined approach

\subsection{Object Count}

First method to determine the density of the lane is to count exact number of vehicles in the lane.To count number of vehicles in the image first thresholds the image ,by converting the grayscale image into an image that contains only two colours. It assigns black colour to all the pixels that have luminosity than a threshold level and the others as white. Approximately calculates the threshold of the image and then complement the image by using this, white patches get converted into black and vice versa. Find the boundaries of the objects and display number of vehicles in the image.

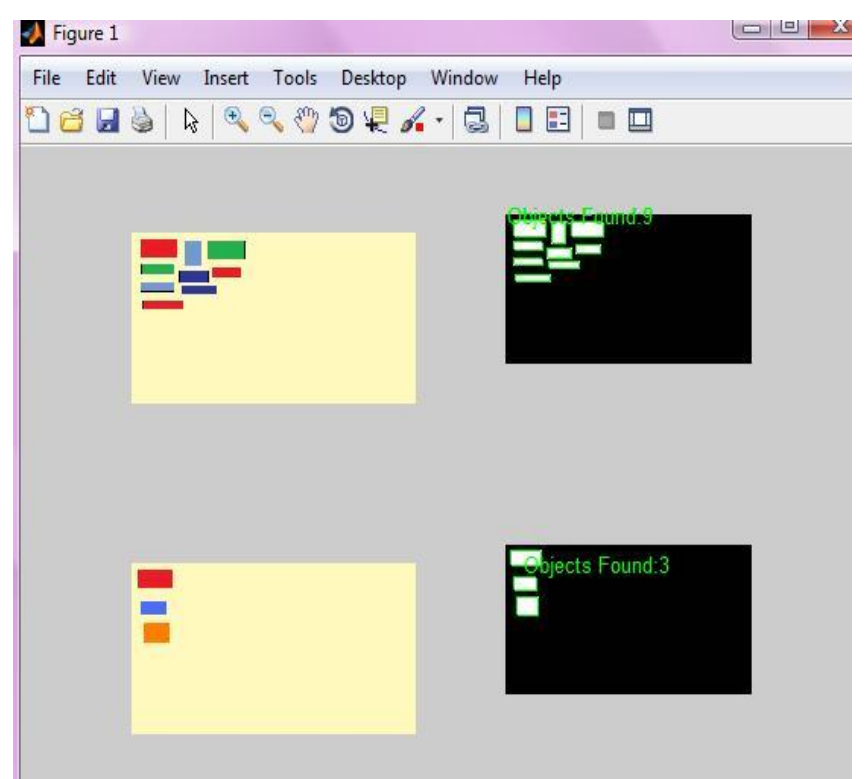

Fig 3.2: Figure demonstrating a checkpoint and objects on lanes to be counted.

\subsection{Queue Length Estimation}

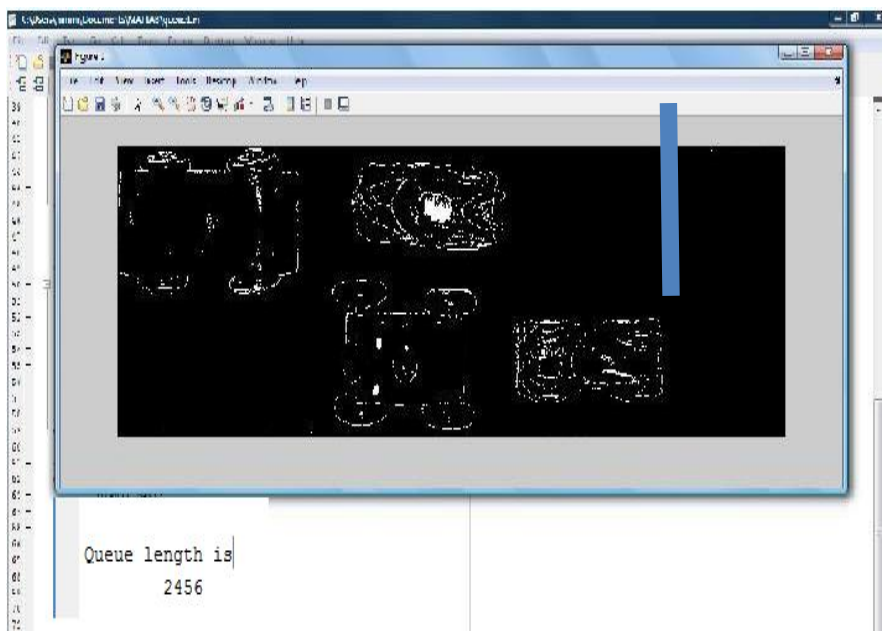

Fig 3.3: Figure showing vehicles on a checkpoint to estimate queue length.

Second method to determine the density of the lane is to estimate the queue length of the lane by approximating the continuity of the vehicles on the particular lane. The images captured from the camera undergo edge detection so that the edges of the vehicles stand out and the image becomes a mixture of black and white pixels. Black stands for the background and white pixels represent the edges of the vehicles. Calculate the continuity of the white pixels in every row till the last. Continue this method till the minimum size of the vehicle is taken into consideration. The moment we realize that white pixels are no more available or their count is very less in that row, we stop the process and declare the queue length.

\subsection{Combined Approach}

In queue length method, the results are quick but not precise as we make use of approximation. While in case of object counting, the results are not as quick as queue length method. So in order to make the tradeoff between accuracy and time required, combined approach is taken into consideration. This approach is combination of the object counting and the queue length estimation method. In some cases, the length of the vehicles is large and hence they occupy more space on the road. Thus, the queue length estimation in these cases is large in spite of less number of vehicles. Hence we combine both the techniques to estimate the density of the vehicles. Consider image in which there is one vehicle in lane 1 but its queue length is 1000 . Consider another lane in which there are 8 vehicles but the queue length is 800 . So by queue length method first lane should be given priority and by object counting lane 2 . In the combined approach, it will check first using queue length the length and then apply object counting, if the count of the vehicles is greater than 5 , then release the vehicle on the lane otherwise check the other lane with the second highest queue length. 


\section{EXPERIMENTAL RESULTS}

Experiment is conducted on 10 different scenarios on 2 different checkpoints and their CPU time was calculated.

\subsection{Queue Length Estimation}

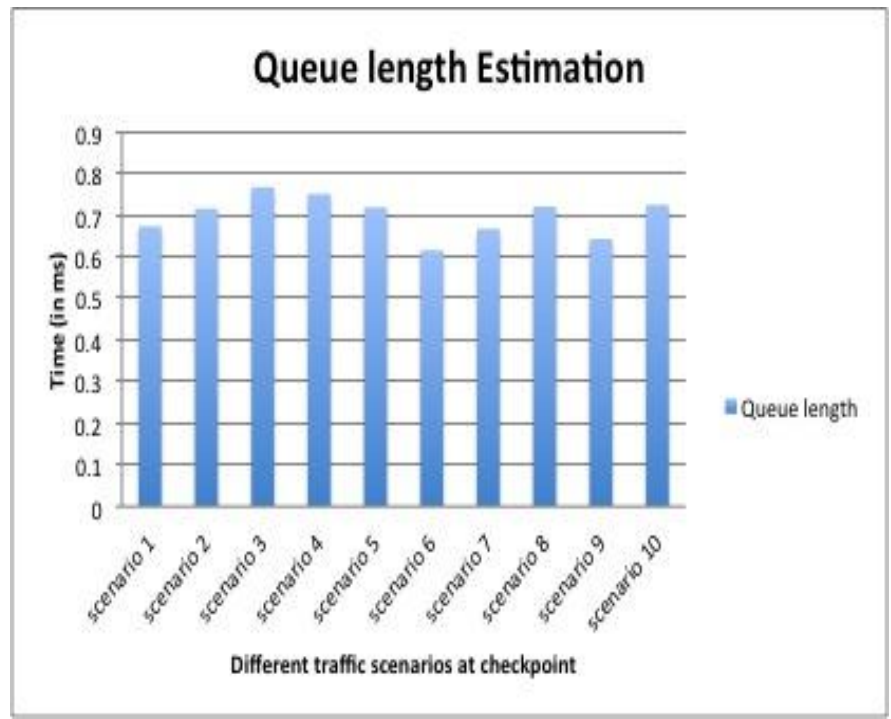

Fig. 4.1 Graph showing queue length estimation

According to the Fig 4.1, it can be seen that the ranges of CPU time in $\mathrm{ms}$ is $0-1$ mainly between 0.6 and 0.8 .

\subsection{Object Counting Method}

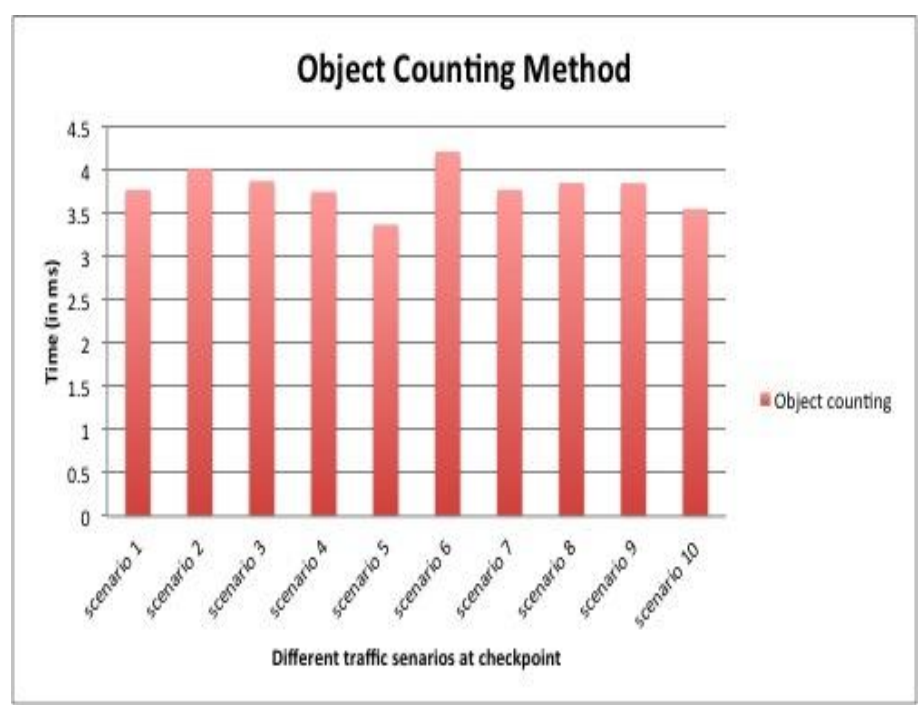

Fig. 4.2 Graph showing object counting method

According to Fig 4.2, it can be seen that the ranges of CPU time in $\mathrm{ms}$ is $3-4.5$.

\subsection{Queue length vs Object Counting vs combined}

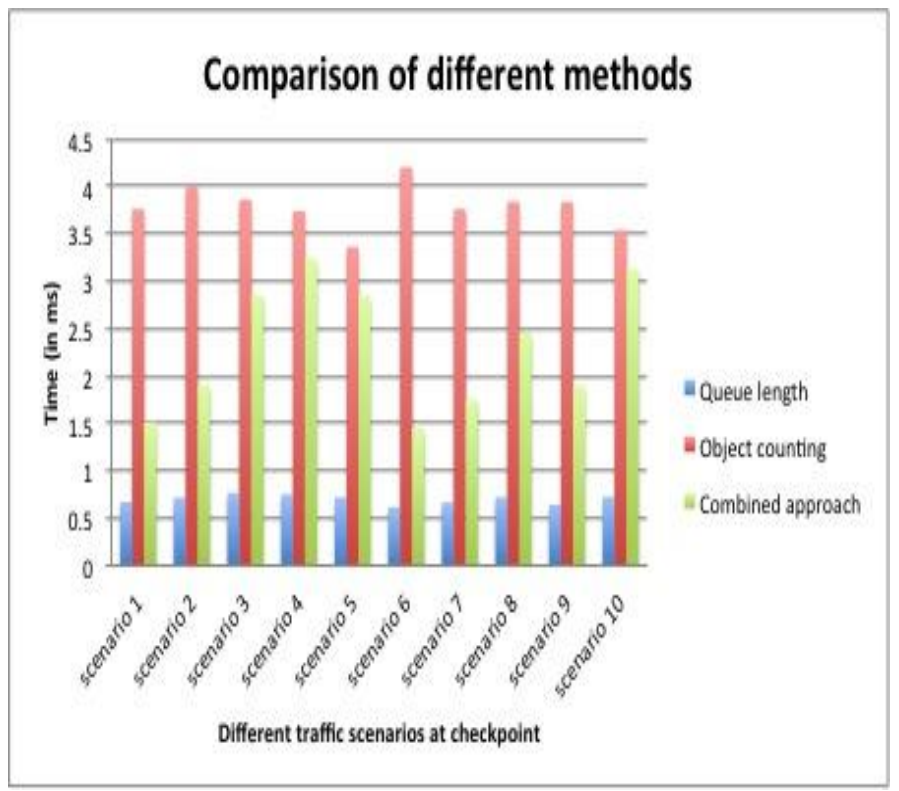

Fig. 4.4 Graph showing comparison between three approaches

According to the Fig 4.4, it is evident that Queue length takes the minimum time. Whereas object Counting takes the maximum time. In the best case scenario, the combined approach takes values in the range 1.5 and hence can be considered as a safe approach.

Table 1: CPU time for all three techniques

\begin{tabular}{|c|c|c|c|}
\hline \multirow[b]{2}{*}{ Scenario } & \multicolumn{3}{|c|}{$\begin{array}{l}\text { Time count for Different techniques (in } \\
\text { ms) }\end{array}$} \\
\hline & $\begin{array}{l}\text { Queue } \\
\text { length } \\
\text { estimation }\end{array}$ & Object count & $\begin{array}{l}\text { Combined } \\
\text { approach }\end{array}$ \\
\hline Scenario 1 & 0.67 & 3.76 & 1.52 \\
\hline Scenario 2 & 0.71 & 4 & 1.9 \\
\hline Scenario 3 & 0.77 & 3.86 & 2.85 \\
\hline Scenario 4 & 0.75 & 3.74 & 3.24 \\
\hline Scenario 5 & 0.71742 & 3.36 & 2.835 \\
\hline Scenario 6 & 0.61436 & 4.2 & 1.452 \\
\hline Scenario 7 & 0.66584 & 3.76 & 1.745 \\
\hline Scenario 8 & 0.71943 & 3.839 & 2.456 \\
\hline Scenario 9 & 0.64174 & 3.836 & 1.897 \\
\hline $\begin{array}{l}\text { Scenario } \\
10\end{array}$ & 0.72336 & 3.539 & 3.123 \\
\hline
\end{tabular}

The table 1 shows the result of the time taken by the three methods to calculate the traffic density. From the table it is evident that queue length estimation takes the least amount of time with values within the range $0-1$, whereas object counting takes the maximum amount of CPU time with values in the range 3-4.The combined approach takes values in the range 1-3.5 and can be considered as safe approach. 


\section{CONCLUSION}

This paper successfully implemented the queue length estimation algorithm, object counting algorithm and edge detection algorithm for estimating the density of traffic on the lanes. The study showed that queue length is a better technique to control the state change of the traffic light as compared to object counting. It shows that it can reduce the traffic congestion and avoids the time being wasted by a green light on an empty road. It visualizes the reality so it functions much better and it consumes the least amount of time as compared to object counting. Overall, the system is good but it still needs improvement to achieve a hundred percent accuracy.

\section{REFERENCES}

[1]. Ahmed S. Salama, Bahaa K. Saleh, Mohamad M. Eassa, "Intelligent Cross Road Traffic Management System (ICRTMS)," 2nd Int. Conf. on Computer Technology and Development, Cairo, Nov 2010, pp. 27-31.

[2]. Somashekhar G.C., S. Shirabadagi, R.S. Hegadi, "High Density traffic Management Using Image Background Subtraction Algorithm", International Journal of Computer Applications (0975 - 8887) Recent Advances in Information Technology, 2014

[3]. N.P. Bhensadadiya, D.Bosamiya,"Survey On Various Intelligent Traffic Management Schemes For Emergency Vehicles", International Journal on Recent and Innovation Trends in Computing and Communication,. 1(11).

[4]. Intelligent Traffic Signal Control System Using Embedded System by Dinesh Rotake and Prof. Swapnili Karmore, Innovative Systems Design And Engineering, ISSN 2222-1727 (paper) ISSN 2222-2871 (online), Vol. 3, No. 5, 2012.

[5]. Priority Based Traffic Lights Controller Using Wireless Sensor Networks by Shruthi K R and Vinodha K, International Journal Of Electronics Signals And Systems (IJESS) ISSN: 2231- 5969, Vol-1 Iss-4, 2012.

[6]. Road Traffic Congestion Monitoring and Measurement using Active RFID and GSM Technology by Koushik Mandal, Arindam Sen, Abhijnan Chakraborty and Siuli Roy, IEEE Annual Conference on Intelligent Transportation Systems, 2011. 